Journal of Quantitative Spectroscopy \&

Radiative Transfer 77 (2003) 23-42
Journal of Quantitative

Spectroscopy \&

Radiative

Transfer

www.elsevier.com/locate/jqsrt

\title{
The $22 \mathrm{GHz}$ radio-aeronomy receiver at Onsala Space Observatory
}

\author{
Peter Forkman ${ }^{\mathrm{a}, *}$, Patrick Eriksson ${ }^{\mathrm{b}}$, Anders Winnberg ${ }^{\mathrm{a}}$ \\ ${ }^{a}$ Onsala Space Observatory, Chalmers University of Technology, S-439 92 Onsala, Sweden \\ ${ }^{\mathrm{b}}$ Department of Radio and Space Science, Chalmers University of Technology, S-412 96 Göteborg, Sweden
}

Received 28 February 2002; accepted 17 May 2002

\begin{abstract}
We present a radiometer system for regular long-term measurements of water vapour in the middle atmosphere. To be able to do continuous and long-term measurements a simple, robust, reliable and automatic system is needed. Our system therefore is based on a stable, uncooled, HEMT amplifier frontend and on a digital spectrometer backend. In order to minimise reflections in the frontend transmission line, which distort the signal due to standing waves, we have designed a corrugated receiver horn, which combines good characteristics (low return loss and sidelobes) and narrow beamwidth to simplify the receiver optics. In order to make the radiometer system as simple as possible, we use the sky as the calibration cold load. This is possible since we use the observed brightness temperatures of an already existing broadband dual-channel $21.0 / 31.4-\mathrm{GHz}$ radiometer, at the observation site, to estimate the brightness temperature of the sky at $22.23 \mathrm{GHz}$. However, we have developed a calibration method, which makes it possible to estimate the sky brightness temperature even if we cannot use the dual-channel radiometer. Despite new measurements, which became available in recent years, the determination of middle atmospheric water vapour distribution still remains a challenge due to the fact that there is a large dispersion among the different measurement methods and data sets, which are obtained on a sparse and sporadic basis. This is the reason why several instruments similar to ours currently are developed in Europe.
\end{abstract}

(C) 2002 Elsevier Science Ltd. All rights reserved.

Keywords: Remote sensing; Microwaves; Radiometers; Measuring techniques; Water vapour; Middle atmosphere; Radiative transfer

\section{Introduction}

Water vapour enters the middle atmosphere primarily by means of the vertical extension of the tropical Hadley cells. When the air passes through the cold tropical tropopause most of the water

\footnotetext{
${ }^{*}$ Corresponding author.

E-mail address: forkman@oso.chalmers.se (P. Forkman).
} 
vapour condenses and freezes. In the stratosphere the water vapour mixing ratio increases with altitude probably due to the oxidation of methane. At about $60 \mathrm{~km}$, nearly all of the methane has been converted into water vapour, producing a water vapour mixing ratio peak of about $7 \mathrm{ppm}_{\mathrm{v}}$. Photo-dissociation due to absorption of Lyman $\alpha$ photons results in a decreasing mixing ratio above $60 \mathrm{~km}$. Transport and photo-dissociation therefore primarily determine the mixing ratio above this altitude. Since the photo-dissociation rate is reasonably well understood, measurements of the water vapour distribution above $60 \mathrm{~km}$ can be used to determine the time scales of mesospheric transport.

Measurements of water vapour can provide important information about 'odd-hydrogen' catalysed ozone destruction and about transport mechanisms in the middle atmosphere. From the middle atmosphere water vapour distribution, dynamical phenomena such as meridional transport, stratospheretroposphere exchange and vortex containment can be investigated due to the long chemical lifetime with respect to the dynamical time scales. Additionally, $\mathrm{H}_{2} \mathrm{O}$ plays a significant role in stratospheric chemistry since it is the major component of the hydrogen content in the middle atmosphere and acts as the primary source of the very reactive $\mathrm{OH}$ radical.

There are several techniques available for measurement and study of middle atmospheric species. In the last decades, radio techniques at millimetre wavelengths have developed into a powerful tool in aeronomy research. The method has been successfully used to detect a number of middle atmospheric molecules including carbon monoxide, ozone, water vapour and chlorine monoxide and to estimate the vertical profile of these molecules.

This technique makes it possible to perform spectral measurements of pure rotational lines both from the ground and from space. These lines are primarily pressure broadened in the middle atmosphere, and therefore the shape of the line profile contains information on the abundance of the emitting constituent as a function of pressure, or equivalently, altitude. This information can be retrieved using mathematical inversion techniques [1].

The two major advantages in using microwaves are the relative insensitivity to aerosol scattering and uncertainties in atmospheric temperature at these wavelengths. At shorter observing wavelengths, aerosol scattering, particularly in the troposphere, must be corrected for. As the size of such aerosols is much smaller than the microwave wavelengths, neither aerosol scattering nor emission has a significant impact on microwave measurements. The thermal emission at microwave wavelengths depends linearly on the temperature (Rayleigh-Jeans approximation) but the strong temperature dependence of infrared emission through the Planck source function requires simultaneous and accurate $(1 \mathrm{~K})$ measurements of atmospheric temperature.

Radio-aeronomical measurements from the sea level, as in the case of our site, of lines originating from the middle atmosphere are possible for frequencies up to about $150 \mathrm{GHz}$. At higher frequencies the troposphere is fairly opaque due to the absorption by water vapour and the lines observed are highly attenuated. In the frequency range $20-150 \mathrm{GHz}$, it is possible to detect carbon monoxide, $\mathrm{CO}(115.3 \mathrm{GHz})$, ozone, O3 (approximately 20 different lines, for example 110.8 and $142.2 \mathrm{GHz}$ ) and water vapour, $\mathrm{H}_{2} \mathrm{O}(22.2 \mathrm{GHz})$. The latter line is possible to study only because it is the result of a transition between high-energy states with relatively scarce populations. The magnetic-dipole transitions of oxygen $\left(\mathrm{O}_{2}\right)$, however, are giving rise to lines in the range $50-70 \mathrm{GHz}$ and at $119 \mathrm{GHz}$, which are completely optically thick when measured from the ground due to the high abundance of oxygen and to the relatively low energies involved. 
Above the altitude at which Doppler broadening begins to exceed pressure broadening as the dominant line broadening mechanism, altitude profiles cannot be calculated. For the 22.2-GHz water vapour line, this transition occurs at about $85-90 \mathrm{~km}$.

The abundance profile can in principle be calculated down to an altitude at which the pressure broadened half-width of the line is equal to the instrument bandwidth. In practice, however, the receiver system, to some degree, distorts the signal. This distortion is seen as a 'baseline ripple' in the measured spectrum. Due to these artefacts in the spectrum it is not always possible to retrieve line information from the whole bandwidth used.

Ground-based radio-aeronomical measurements have provided data on water vapour in the middle atmosphere and have proven to be especially successful in measurements of the mesosphere. See for example Nedoluha et al. [2-4] and Seele and Hartogh [5,6].

This paper is divided into five sections. Section 1 deals with the design of the receiver system, the fundamentals of measurements and calibration, the relation between brightness temperature and antenna temperature, the determination of the optimal elevation angle of measurement and the calibration of the elevation scale. Section 2 is devoted to the troposphere and how one can determine its zenith brightness temperature and its optical depth for correction of the middle atmosphere water vapour line signal. Section 3 gives a brief description of the procedures followed during the measurements. In Section 4 the sources for deformations of the spectral baseline (gain instabilities and reflections) are presented and methods for their mitigation are suggested. Finally, a summary of salient points of the paper is given in Section 5 .

\section{The receiver system, calibration and measuring principles}

\subsection{The receiver system}

The water vapour receiver station consists of a spectral, Dicke-switched, heterodyne receiver for the $6_{16} \rightarrow 5_{23}, 22.23508 \mathrm{GHz}$ transition in $\mathrm{H}_{2} \mathrm{O}$ based on an uncooled HEMT preamplifier (Figs. 1 and 2 and Table 1).

The spectrometer is an analogue filter-digital correlator hybrid spectrometer designed and built at Onsala Space Observatory. The spectrometer uses a small number of filters to divide up the large bandwidth into $20-\mathrm{MHz}$ bands and each filter is then connected to a 2-bit autocorrelator. The total number of correlator delay channels is 800 and the possibility to assign different numbers of correlator channels to different filters makes it possible to operate the spectrometer in six different configurations from $20 \mathrm{MHz}$ bandwidth with a nominal resolution of $25 \mathrm{kHz}$ to $640 \mathrm{MHz}$ bandwidth with a nominal resolution of $800 \mathrm{kHz}$ in steps of factors of 2 [7].

The computer controls and reads off the data from the spectrometer and performs Fast Fourier Transforms to calculate the power spectrum from the measured autocorrelation function. In addition it steers the movable loads and it reads off temperature sensors, phaselock sensors and the weather station of the observatory.

\subsection{Measurement principles}

To account for the collection of thermal noise power by the antenna, we introduce the antenna, temperature $T_{\mathrm{a}}=P / k$ where $P$ is the power per unit bandwidth and $k$ is Boltzmann's constant. 


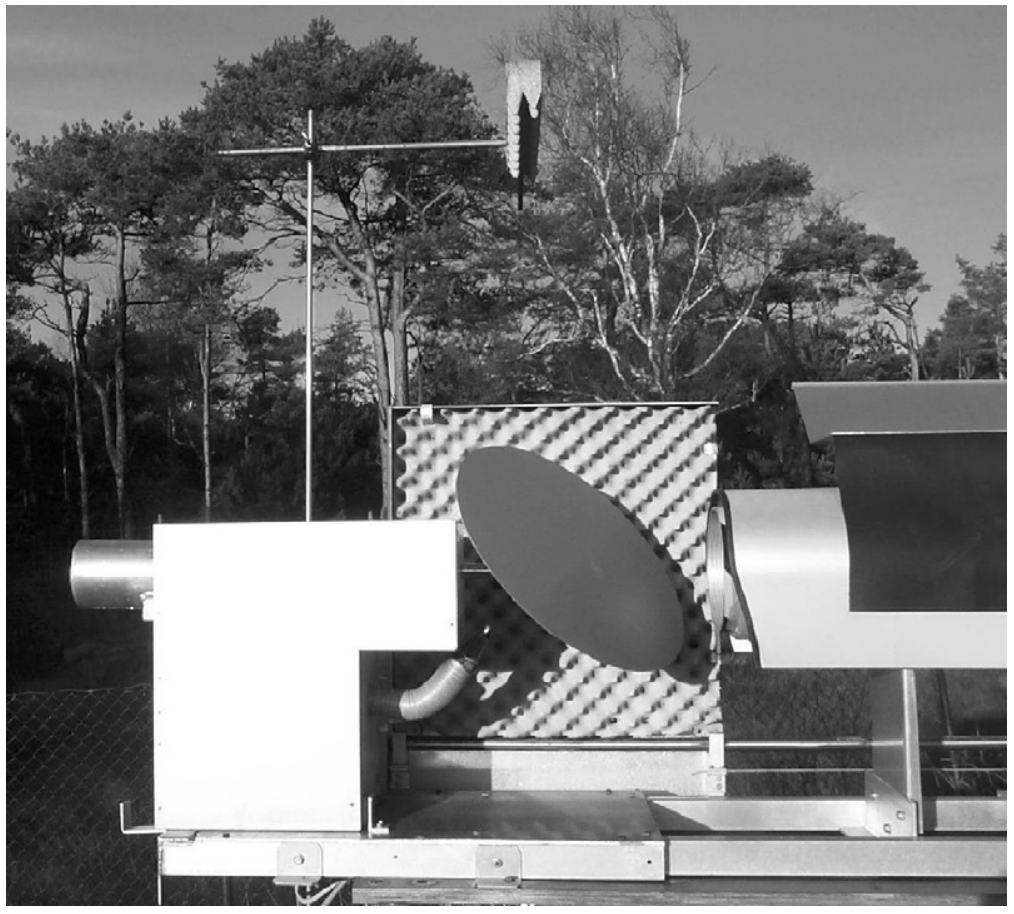

Fig. 1. The receiver during the calibration cycle when the beam is switched, by the mirror, between the cold load (the sky at $45^{\circ}$ elevation) and the hot load (the absorber behind the mirror). In the measuring mode the beam is switched between the signal direction, the sky at $\sim 20^{\circ}$ elevation, and the reference direction, the zenith sky. The piece of absorber in the reference beam balances the system by compensating for the higher system noise level in the signal beam.

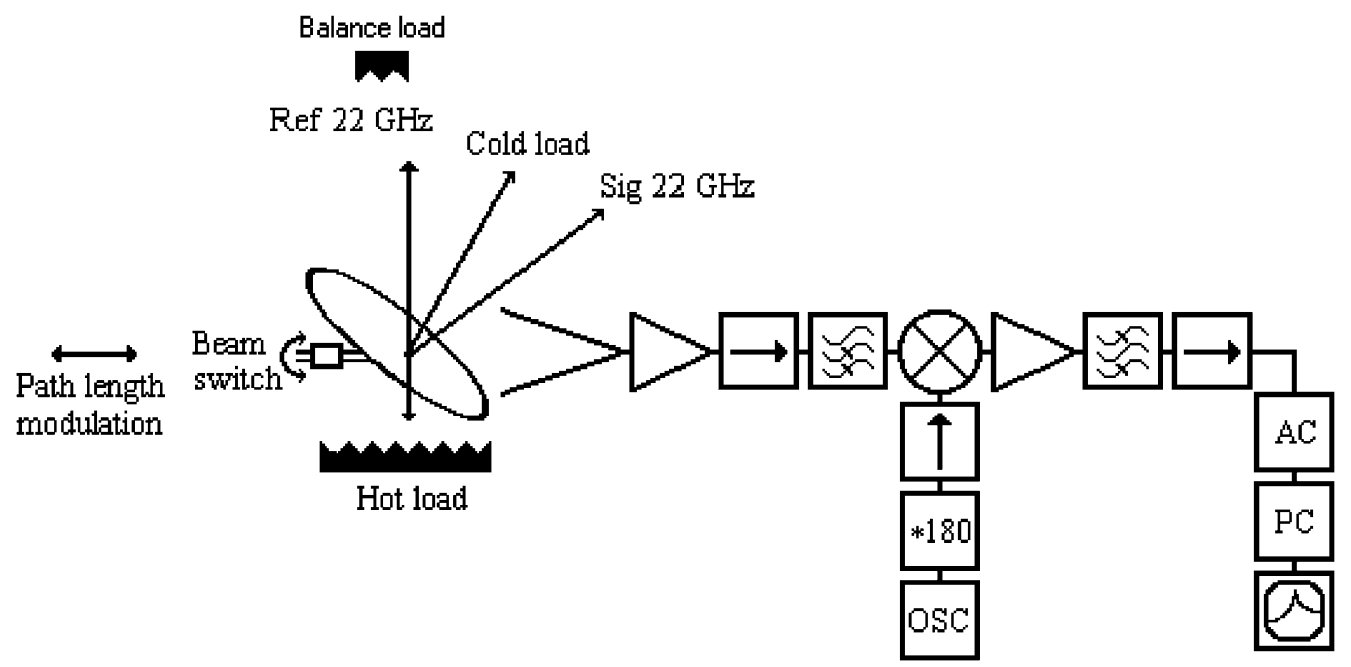

Fig. 2. A block diagram of the receiver. $\mathrm{AC}$ is the digital autocorrelator, $\mathrm{PC}$ is the master computer and $\mathrm{OSC}$ is the local oscillator feed $(\sim 100 \mathrm{MHz})$ to the $180 \times$ multiplier. 
Table 1

Technical receiver specifications

\begin{tabular}{|c|c|c|c|}
\hline Parameter & Value & Parameter & Value \\
\hline Radio frequency & $21.2-23.6 \mathrm{GHz}, \mathrm{SSB}$ & $\begin{array}{l}\text { Mixer IF } \\
\text { Conversion loss }\end{array}$ & $\begin{array}{l}4.0 \pm 0.7 \mathrm{GHz} \\
<5 \mathrm{~dB}\end{array}$ \\
\hline $\begin{array}{l}\text { Mirror coverage } \\
\text { Turning angle resolution }\end{array}$ & $\begin{array}{l}-35 \mathrm{~dB} \text { (Gaussian beam approx.) } \\
\pm 0.3^{\circ}\end{array}$ & Manufacturer & SPACEK lab. \\
\hline Elevation accuracy & $\pm 0.5^{\circ}$ & $\begin{array}{l}\text { Local oscillator } \\
\text { Output frequency }\end{array}$ & $\begin{array}{l}\text { Phaselocked freq. multiplier } \\
18.229 \mathrm{GHz}\end{array}$ \\
\hline Horn & Aluminium, corrugated & Output power & $17 \mathrm{dBm}$ \\
\hline $3 \mathrm{~dB}$ full beamwidth & $6^{\circ}$ (see Fig. 3) & Manufacturer & MITEQ \\
\hline Return loss & $-35 \mathrm{~dB}$ & & \\
\hline Total horn length & $806 \mathrm{~mm}$ & IF amplifier gain & $50 \mathrm{~dB}$ \\
\hline Aperture radius & $95 \mathrm{~mm}$ & Noise temperature & $110 \mathrm{~K}$ \\
\hline Corrugation period/width/tooth & $4 / 3 / 1 \mathrm{~mm}$ & Manufacturer & MITEQ \\
\hline Number of corrugations & 186 & Bandpass filter $-1 \mathrm{~dB}$ & $3.35-4.65 \mathrm{GHz}$ \\
\hline Preamplifier & Uncooled HEMT & Return loss & $-14 \mathrm{~dB}$ \\
\hline Gain & $16 \mathrm{~dB}$ & Manufacturer & $K \& L$ \\
\hline Noise temperature & $235 \mathrm{k}$ & & \\
\hline Manufacturer & SPACEK lab. & $\begin{array}{l}\text { Isolator } \\
\text { Isolation }\end{array}$ & $\begin{array}{l}3.0-5.0 \mathrm{GHz} \\
20 \mathrm{~dB}\end{array}$ \\
\hline Isolator & $21.2-23.6 \mathrm{GHz}$ & Return loss & $-19 \mathrm{~dB}$ \\
\hline Isolation & $18 \mathrm{~dB}$ & Manufacturer & QUEST \\
\hline Return loss & $-17 \mathrm{~dB}$ & & \\
\hline Manufacturer & QUEST & Total receiver temperature & $\approx 250 \mathrm{~K}$ \\
\hline $\begin{array}{l}\text { Lower sideband suppression } \\
\text { Manufacturer }\end{array}$ & $\begin{array}{l}\text { Waveguide filter, }-50 \mathrm{~dB} \\
\text { SPACEK lab. }\end{array}$ & Measurement technique & Balanced skyswitching \\
\hline
\end{tabular}

The receiver output power must be calibrated in units of antenna temperature. The net IF power reaching the square law detector consists of two components. The one that represents the amplified signal is proportional to the antenna temperature, $T_{\mathrm{a}}$. The other component is the power generated internally, which we identify as the receiver temperature, $T_{\text {rec }}$, which has to be calibrated on the same scale as the antenna temperature. The sum of these components is the system temperature $T_{\text {sys }}=T_{\mathrm{a}}+T_{\text {rec }}$.

During the calibration cycle, two blackbody emitters are observed with temperatures $T_{\text {hot }}$ and $T_{\text {cold }}$ and the output signal power from the spectrometer is registered, $P_{\mathrm{a}}=G(v) k\left(T_{\mathrm{a}}+T_{\text {rec }}\right) \Delta v$ where $G$ is the system gain and $\Delta v$ is the bandwidth of the receiver. The normalised difference spectrum is formed:

$$
\frac{P_{\text {hot }}-P_{\text {cold }}}{P_{\text {cold }}}=\frac{T_{\text {hot }}-T_{\text {cold }}}{T_{\text {cold }}+T_{\text {rec }}} .
$$

If we know the physical temperatures and the outputs we can calculate the channel-to-channel receiver temperature,

$$
T_{\text {rec }}=\frac{T_{\text {hot }} P_{\text {cold }}-T_{\text {cold }} P_{\text {cold }}}{P_{\text {hot }}-P_{\text {cold }}},
$$


and

$$
T_{\text {sys }}=T_{\text {cold }}+T_{\text {rec }}=P_{\text {cold }} \frac{T_{\text {hot }}-T_{\text {cold }}}{P_{\text {hot }}-P_{\text {cold }}}
$$

During the observation cycle we observe $\Delta T_{\mathrm{a}}=T_{\text {sig }}-T_{\text {ref }}$,

$$
\Delta T_{\mathrm{a}}=\Delta P \frac{T_{\text {hot }}-T_{\text {cold }}}{P_{\text {hot }}-P_{\text {cold }}},
$$

where $\Delta P=P_{\text {sig }}-P_{\text {ref }}$.

Since the actual temperatures of the hot and cold loads are not known during the calibration $\Delta T_{\mathrm{a}}$ has to be rescaled in the data reduction software.

$$
\Delta T_{\mathrm{a}}^{\text {new }}=\Delta T_{\mathrm{a}}^{\mathrm{old}} \frac{T_{\text {hot }}-T_{\text {cold }}}{T_{\text {hot }}^{\text {def }}-T_{\text {cold }}^{\text {def }}} .
$$

\subsection{Relationship between brightness temperature and antenna temperature}

Using the Rayleigh-Jeans approximation and the equation of radiative transfer the brightness temperature, which is frequency dependent, can be written as follows [8]:

$$
T_{\mathrm{b}}(v)=T_{\mathrm{bg}} \mathrm{e}^{-\tau_{\infty}}+\int_{0}^{\infty} T(s) \mathrm{e}^{-\tau(v, s)} \alpha(v, s) \mathrm{d} s,
$$

where $T_{\mathrm{bg}}$ is the brightness temperature of the background radiation, $T$ is the physical temperature along the path and $\alpha$ is the absorption coefficient due to water vapour, liquid water and oxygen $\left(\alpha=\alpha_{v}+\alpha_{1}+\alpha_{0}\right)$. The parameter $\tau$ is the opacity from the ground to the point at $s$ :

$$
\tau(v, s)=\int_{0}^{s} \alpha(x) \mathrm{d} x
$$

$\tau_{\infty}$ is the total opacity $[\tau(v, \infty)]$. The contribution to $\tau$ is completely dominated by the troposphere. By assuming that the troposphere is horizontally stratified and introducing $T_{\text {eff }}$, the effective temperature of the troposphere, defined as

$$
T_{\text {eff }}=\frac{\int_{0}^{\infty} T(s) \alpha(v, s) \mathrm{e}^{-\tau(v, s)} \mathrm{d} s}{\int_{0}^{\infty} \alpha(v, s) \mathrm{e}^{-\tau(v, s)} \mathrm{d} s},
$$

the sky brightness temperature can be written as

$$
T_{\mathrm{b}}(v)=T_{\mathrm{bg}} \mathrm{e}^{-\tau}+T_{\text {eff }}\left(1-\mathrm{e}^{-\tau}\right),
$$

where $\tau=\tau_{\infty}$. In Section 1.2 the antenna temperature $T_{\mathrm{a}}$ was introduced. However, $T_{\mathrm{a}}$ is a virtual temperature whereas $T_{\mathrm{b}}$ is the real radiation temperature. The relation between these two temperatures has to be investigated.

When the feed horn is directed against the sky the antenna temperature is the sky brightness temperature weighted with the horn-antenna radiation pattern, $F$, since some parts of the main lobe and the side lobes of the antenna 'see' contributions from higher elevations and some parts from 


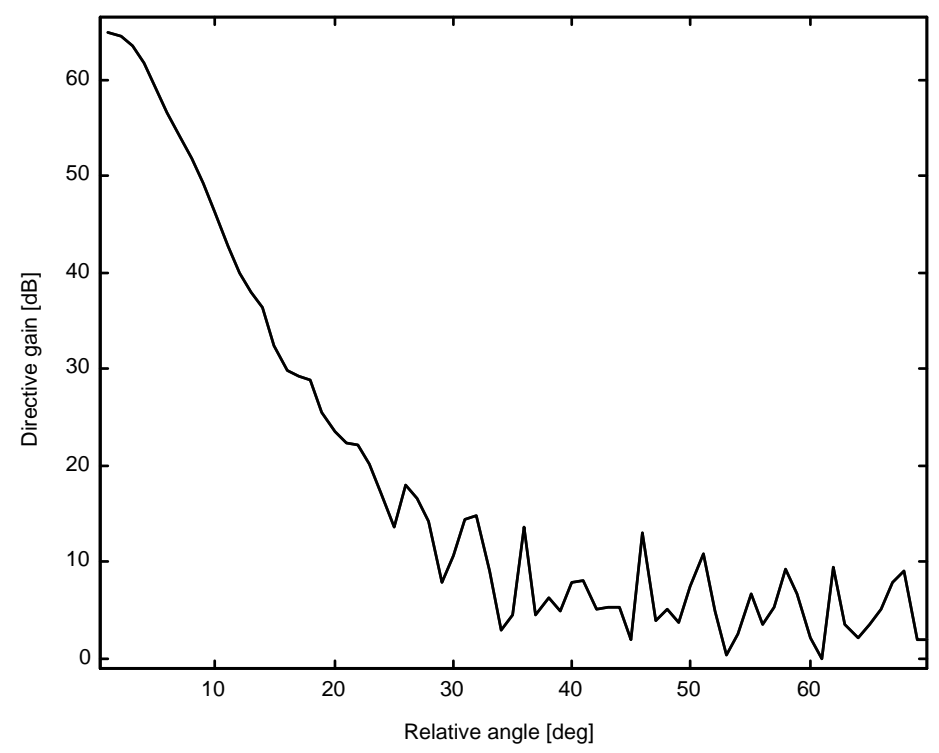

Fig. 3. The measured E-plane radiation pattern for the $22-\mathrm{GHz}$ corrugated feed horn. When the relative angle is less than $30^{\circ}$ the radiation pattern shows the features of the horn. At higher relative angles the sensitivity limit of the analyser is reached.

lower elevations and the ground. The antenna temperature, $T_{\mathrm{a}}$, therefore can be written as

$$
T_{\mathrm{a}}=\frac{\iint_{4 \pi} T_{\mathrm{b}}(\theta, \varphi) F(\theta, \varphi) \mathrm{d} \Omega}{\iint_{4 \pi} F(\theta, \varphi)} \mathrm{d} \Omega,
$$

where $\theta$ and $\varphi$ are the spherical co-ordinates relative to the pointing direction of the antenna and $F(0,0)=1$. It is possible to calculate the so-called coupling efficiency, $\eta=T_{\mathrm{a}} / T_{\mathrm{b}}$, if the antenna radiation pattern, $F(\theta, \varphi)$ (see Fig. 3), and the brightness temperature distribution, $T_{\mathrm{b}}(\theta, \varphi)$, are known. Fig. 4 shows the coupling efficiency, for the troposphere, as a function of elevation angle of the feed horn. For this calculation the effective height of the troposphere has been approximated by $4000 \mathrm{~m}$ and the effective temperature, $T_{\text {eff }}$, by $0.95 T_{\text {ground }}$ (see Section 2). The tropospheric absorption coefficient, $\alpha$, and $T_{\text {ground }}$ have been given typical clear winter day values $\left(\alpha=2.1 \times 10^{-5} \mathrm{~m}^{-1}\right.$ and $T_{\text {ground }}=270 \mathrm{~K}$ ) and the antenna radiation pattern has been assumed to be rotationally symmetric.

$T_{\mathrm{a}}$ is calculated from Eq. (1.9) where $F(\theta, \varphi)$ is taken from Fig. 3. To be able to calculate $T_{\mathrm{b}}(\theta, \varphi)$ from Eq. (1.8) the elevation related to $(\theta, \varphi)$ was calculated and the optical depth of the troposphere was given by $\tau=\alpha s$, where $s$ is the tropospheric path length. The relation, in spherical geometry, between vertical altitude, $h$, and distance from the ground along the path, $s$, can be expressed by the law of cosines which rearranged gives

$$
s=\sqrt{(R+h)^{2}-R^{2} \cos ^{2}(e l)}-R \sin (e l),
$$

where $R$ is the radius of the Earth and $e l$ is the elevation angle. If the elevation related to $(\theta, \varphi)$ is found to be negative $T_{\mathrm{b}}(\theta, \varphi)=T_{\text {ground }}$ (this part of the beam 'sees' the ground). 


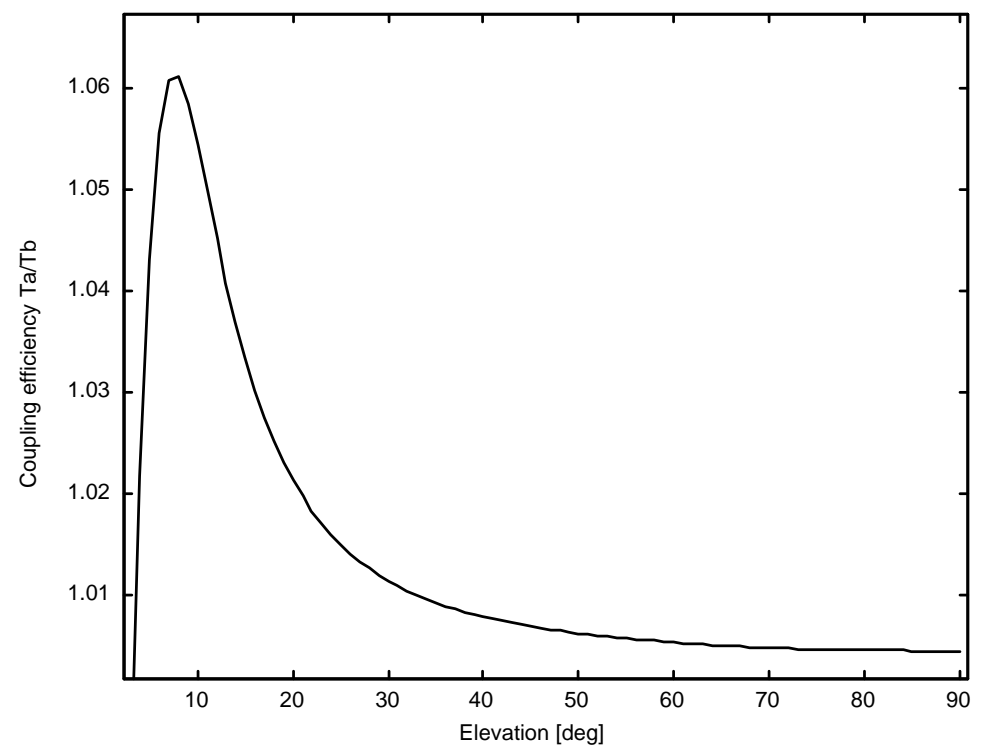

Fig. 4. The coupling efficiency as a function of elevation angle.

The curve, in Fig. 4, at about $10^{\circ}$ is explained by the fact that the lower part of the beam sees more emission than the higher part of the beam. The mean value of these contributions is higher than the sky brightness temperature in the actual elevation since the path length increases non-linearly with lower elevations. Below $10^{\circ}$ the increase in the lower parts of the beam is diminished due to the fact that these parts 'see' the ground instead of a steadily increasing path length in the atmosphere.

In our case the horn is mounted horizontally and a mirror is placed in front of and close to the horn and this mirror turns the main part of the beam $(>-35 \mathrm{~dB})$ to the desired elevation (see Fig. 1). We believe that all of the detectable power is reflected from the mirror since we think that the mirror covers almost the entire near-field radiation pattern. This means that the coupling efficiency for our mounting is the same as if we turned the horn to the desired elevation.

\subsection{Optimal elevation angles}

The elevation dependence of the signal-to-system temperature ratio is investigated in order to choose an elevation angle that gives the highest signal-to-system temperature ratio.

The statistical uncertainty in the measured antenna temperature, $\Delta T_{\mathrm{a}}$, can be expressed by the radiometer noise formula, $\sigma\left(\Delta T_{\mathrm{a}}\right)=K T_{\text {sys }} / \sqrt{B t}$, where $K$ is the noise performance depending on various receiver configurations, $T_{\mathrm{sys}}=T_{\mathrm{a}}+T_{\mathrm{rec}}, B$ is the bandwidth of the noise power and $t$ is the integration time. The major part of $T_{\mathrm{a}}$ is the tropospheric emission which varies between 15 and $250 \mathrm{~K}$ depending on elevation and weather conditions and $T_{\text {rec }} \approx 250 \mathrm{~K}$. The middle atmospheric contribution to $T_{\mathrm{a}}$ (which we want to study) is less than $1 \mathrm{~K}$. At a lower elevation angle a longer path through the middle atmosphere is obtained but the tropospheric attenuation will also increase. At a lower elevation the received tropospheric emission will also increase which in turn increases the statistical uncertainty, $\sigma\left(\Delta T_{\mathrm{a}}\right)$, of the measured antenna temperature. 


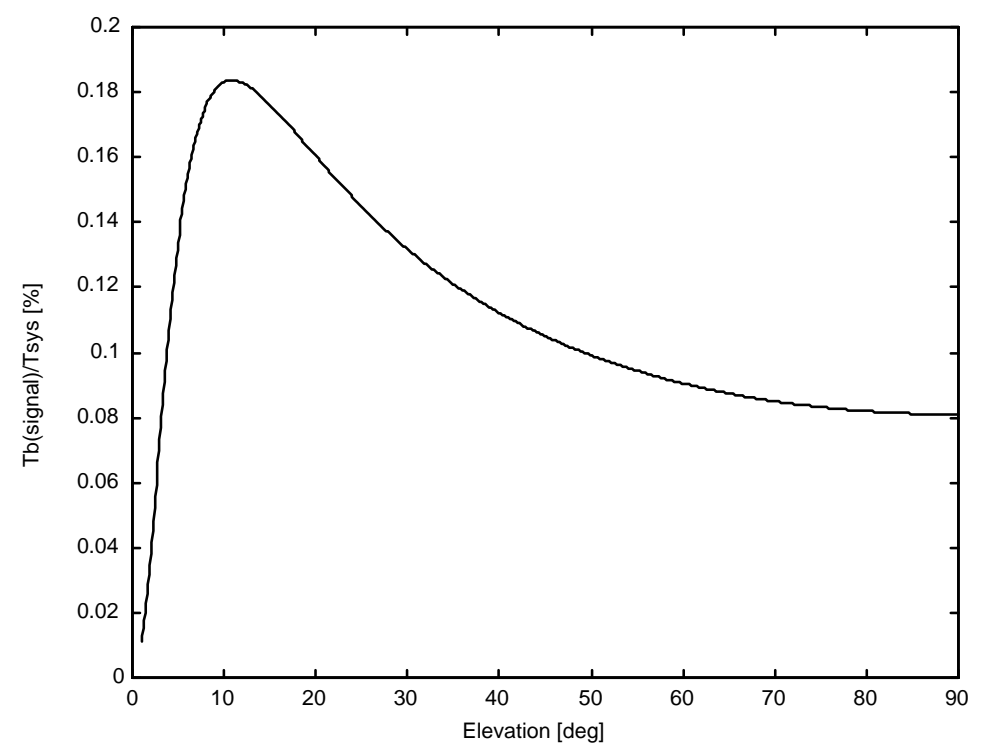

Fig. 5. The ratio between the received middle atmospheric signal and the system temperature (in \%) plotted against the elevation angle. As seen in the figure an elevation angle of about $10^{\circ}$ optimises the observation conditions.

The intensity received at ground level will change as a function of elevation angle due to the exponential relation between path length and absorption/emission. The elevation angle, which gives the highest, received middle atmospheric signal-to-system temperature ratio, optimises the observation conditions.

Fig. 5 shows the calculated ratio between the middle atmospheric signal and the system temperature, as observed from the ground, plotted against elevation angle. In this calculation the atmosphere has been approximated with one tropospheric layer and one 'middle atmospheric layer'. The absorption coefficients and the effective temperatures of the two layers have been chosen to simulate standard observation conditions. It is found that an elevation angle of about $10^{\circ}$ optimises the observation conditions but due to the topography at our observation site we have to use elevation angles $>15^{\circ}$. When $h<10000 \mathrm{~m}$ (as in the troposphere) and the elevation $\mathrm{el}>15^{\circ}, s$ in Eq. (1.10) is well approximated by $h / \sin (e l)$ which is the same as in a flat geometry.

\subsection{Pointing}

The online computer adjusts the elevation of the pointing mirror. The turning angle of the mirror axis is measured with high precision but the turning angle has to be calibrated to the actual elevation. This is done with the aid of a pencil laser that is mounted in the horn throat. When the mirror is turned the laser beam moves along a vertical scale mounted for the calibration.

On clear days it is possible to perform so called dip curve measurements, which means that the sky brightness temperature is measured at a number of different elevation angles. Then it is possible to verify the elevation angle calibration under the assumption that the tropospheric absorption coefficient is the same in all directions. 
Table 2

Technical specifications for the dual-channel water vapour radiometer

\begin{tabular}{|c|c|}
\hline Parameter & Value \\
\hline Frequencies & 21.0 and $31.4 \mathrm{GHz}$ \\
\hline Antenna (one for each frequency) & Dielectrically loaded horn \\
\hline Antenna beam widths & $6^{\circ}$ (both channels) \\
\hline Mount & Azimuth/elevation \\
\hline Positioner & Step motors, $0.025^{\circ} /$ step after gearbox \\
\hline Slew rate & $1.8^{\circ} / \mathrm{s}$ \\
\hline Pointing resolution & $0.1^{\circ}$ \\
\hline Reference load temperatures, cold mode & 77 and $313 \mathrm{~K}$ \\
\hline Reference load temperatures, hot mode & 313 and $360 \mathrm{~K}$ \\
\hline System noise temperature $21.0 \mathrm{GHz}$ & $\sim 450 \mathrm{~K}$ \\
\hline System noise temperature $31.4 \mathrm{GHz}$ & $\sim 550 \mathrm{~K}$ \\
\hline RF bandwidth (both channels) & $\sim 1 \mathrm{GHz}$ \\
\hline Accuracy & $<1 \mathrm{~K}$ \\
\hline
\end{tabular}

The balance load adds the same emission to the reference beam in all measurements but the tropospheric opacity changes slightly from measurement to measurement and therefore there is a need for a fine adjustment to keep the emission the same in the signal beam as in the reference beam. This is done by slightly changing the signal elevation angle for every new measurement. After each measured spectrum the computer calculates a new signal elevation angle, from the brightness temperature in the measured difference (signal - reference) spectrum to be used in the next measurement. If the offset in the difference spectrum is positive there is more emission in the signal beam than in the reference beam and the next signal elevation is therefore corrected to a higher elevation. It is possible to use a linear relationship between the measured difference spectrum and the needed elevation change if the variation in the tropospheric opacity is slow. The estimation of the proportional constant can be improved by studying the history of several measured spectra to the commanded elevation changes.

\section{Determination of the tropospheric opacity}

In order to use the sky as a cold load for calibration, simultaneous data from the tropospheric water vapour dual-channel radiometer placed at the observatory are used. This radiometer monitors the integrated amounts of tropospheric water vapour and liquid water and it operates at 21.0 and $31.4 \mathrm{GHz}$ (Table 2) [9].

\subsection{Estimation of the zenith sky brightness temperature}

To be able to calculate the zenith sky brightness temperature at $22.23 \mathrm{GHz}, T_{\mathrm{b}}\left(22.2 \mathrm{GHz}, 90^{\circ}\right)$, from the outputs of the dual channel radiometer, the zenith brightness temperatures $T_{\mathrm{b}}\left(21 \mathrm{GHz}, 90^{\circ}\right)$ and $T_{\mathrm{b}}\left(31 \mathrm{GHz}, 90^{\circ}\right)$, relations between these different brightness temperatures have to be found. 


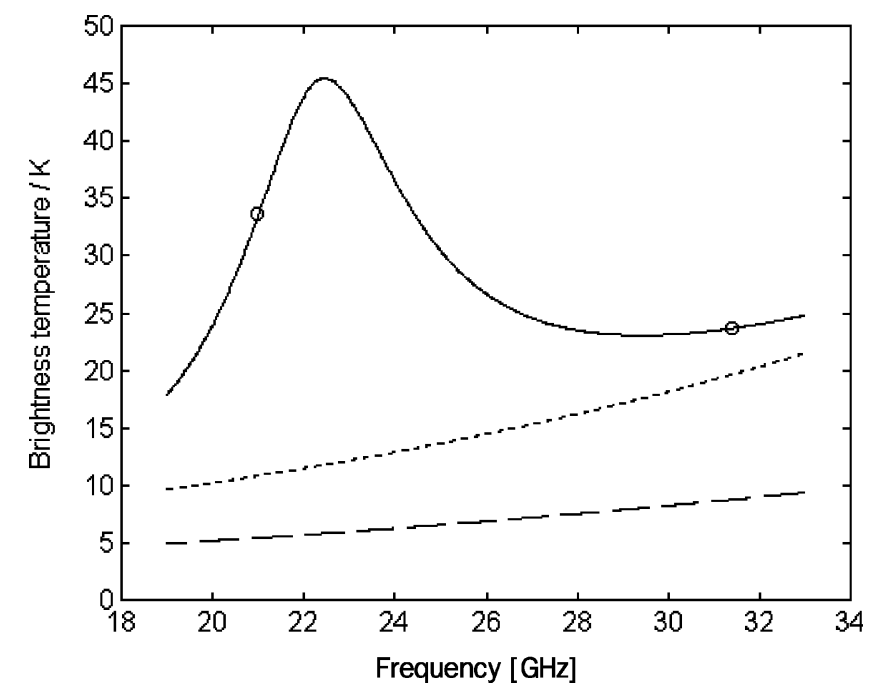

Fig. 6. A synthetic spectrum of the sky brightness temperature, as seen from the ground. The dashed line includes the contribution from the cosmic background and liquid water. The dotted line also includes $\mathrm{O}_{2}$ and transitions at frequencies higher than $22 \mathrm{GHz}$ in $\mathrm{H}_{2} \mathrm{O}$. The solid line, finally, also includes the 22-GHz $\mathrm{H}_{2} \mathrm{O}$ transition. The circles mark the 21.0/31.4 GHz frequencies.

We therefore need to estimate the polynomial function $f_{1}$ as defined by

$$
T_{\mathrm{b}}\left(22.2 \mathrm{GHz}, 90^{\circ}\right)=f_{1}\left[T_{\mathrm{b}}\left(21 \mathrm{GHz}, 90^{\circ}\right), T_{\mathrm{b}}\left(31 \mathrm{GHz}, 90^{\circ}\right)\right] .
$$

As seen in Fig. 6 the correlation between $T_{\mathrm{b}}\left(22.2 \mathrm{GHz}, 90^{\circ}\right)$ and $T_{\mathrm{b}}\left(21 \mathrm{GHz}, 90^{\circ}\right)$ is expected to be high but since the influence from other lines $\left(\mathrm{O}_{2}\right.$ and $\mathrm{H}_{2} \mathrm{O}$ at higher frequencies $)$ and from liquid water increases with frequency $T_{\mathrm{b}}\left(31 \mathrm{GHz}, 90^{\circ}\right)$ is also included in the polynomial fit.

To be able to estimate $f_{1}, 1$ year of radiosonde data from the Gothenburg-Landvetter Airport (37 km north-east of the observatory) have been used together with a forward model software, developed by Jarlemark [10]. The forward model uses a millimetre-wave propagation model [11] to calculate the absorption coefficients for oxygen, water vapour and the atmospheric liquid water content (clouds). Information on the highly variable liquid water density is needed to calculate the attenuation due to clouds. Since the radiosonde just gives us temperature, pressure and humidity as a function of height a cloud model to simulate the amount of liquid water is needed. A cloud is formed by a rising parcel of moist air passing through and above a level whose temperature is such that the relative humidity of the parcel reaches $100 \%$. The water vapour content at this point is determined from the saturation density $(\rho)$. As the air continues to rise, the temperature decreases, and hence the saturation density decreases. The difference between $\rho$ at the cloud base and at the cloud top, $\rho_{\text {base }}-\rho_{\text {top }}$, is a measure of the condensed water vapour of a rising air parcel, which is called the adiabatic liquid water content.

To estimate the water content within a cloud the adiabatic liquid water content can be multiplied by a so-called tuning factor depending on the various cloud types [12]. We use a similar but empirically estimated cloud model [13], to find the liquid water density as a function of cloud thickness. 
Polynomial functions of different degrees were tested by applying the method of least squares to the processed radiosonde data and finally a polynomial of the first degree was adopted

$$
T_{\mathrm{b}}\left(22.2 \mathrm{GHz}, 90^{\circ}\right)=c_{0}+c_{1} T_{\mathrm{b}}\left(21 \mathrm{GHz}, 90^{\circ}\right)+c_{2} T_{\mathrm{b}}\left(31 \mathrm{GHz}, 90^{\circ}\right),
$$

where the constants $c_{0}=-1.11, c_{1}=1.49$ and $c_{2}=-0.19$ are found. Errors in the estimations of $c_{0}, c_{1}, c_{2}$ and in the measured $T_{\mathrm{b}}\left(21 \mathrm{GHz}, 90^{\circ}\right)$ and $T_{\mathrm{b}}\left(31 \mathrm{GHz}, 90^{\circ}\right)$ gives $\sigma\left[T_{\mathrm{b}}\left(22.2 \mathrm{GHz}, 90^{\circ}\right)\right]$ $\approx 2 \mathrm{~K}$.

Our estimation of the weighted mean tropospheric temperature, $T_{\text {eff }}$, is based on the correlation between $T_{\text {eff }}$ and the ground temperature, $T_{\text {ground }}$. To try to make the model even more accurate the zenith sky brightness temperature was also included in the model:

$$
T_{\text {eff }}(22.2 \mathrm{GHz})=d_{1} T_{\text {ground }}+d_{2} T_{\mathrm{b}}\left(22.2 \mathrm{GHz}, 90^{\circ}\right),
$$

where $d_{1}$ and $d_{2}$ are found to be 0.948 and 0.048 , respectively.

The correlation between $T_{\text {eff }}$ and $T_{\text {ground }}$ is high, as expected. Information about the brightness temperature at $22 \mathrm{GHz}$ contributes with about $1 \%$ to the obtained value of $T_{\text {eff }}$. Errors in the estimations of $d_{1}, d_{2}$ and $T_{\text {ground }}$ gives $\sigma\left[T_{\text {eff }}(22.2 \mathrm{GHz})\right] \approx 3 \mathrm{~K}$.

Calibrating the 22.2-GHz receiver with two known blackbody loads and then comparing the measured brightness temperature of the zenith sky with the estimated zenith brightness temperature from the 21.0/31.4-GHz receivers checks the accuracy of the models. These comparisons indicate that the obtained results lie within the expected errors.

The middle atmospheric $22.23-\mathrm{GHz}$ transition is, of course, seen in the $T_{\text {cold }}$ direction and this introduces a systematic error of about $1 \%$ in the observed $\Delta T_{\mathrm{b}}$. This error and the slope of the sky brightness temperature within the frequency range (due to the tropospheric water content, see Fig. 6) can be included to increase the accuracy of the $T_{\text {cold }}$ estimation.

Similar methods to estimate the sky brightness temperature and the effective temperature of the troposphere, for different frequencies, by the help of a dual channel water vapour radiometer are discussed by Ingold et al. [14] and by Peter and Kaempfer [15].

\subsection{Corrections for the tropospheric attenuation at $22.2 \mathrm{GHz}$}

In the following section the relation between vertical altitude, $h$, elevation, $e l$, and the distance from the ground along the path, $s$, is approximated by $s=h / \sin (e l)$ (flat geometry see Sections 1.3 and 1.4) because the observing elevation is $\sim 20^{\circ}$.

From the dual channel radiometer and the weather station we get $T_{\mathrm{b}}\left(90^{\circ}\right)$ and $T_{\text {eff. It }}$ is now possible to calculate the opacity of the troposphere, $\tau$, at one air mass (zenith direction):

$$
\tau=-\log \left(\frac{T_{\text {eff }}-T_{\mathrm{b}}\left(90^{\circ}\right)}{T_{\mathrm{eff}}-T_{\mathrm{bg}}}\right) .
$$

The sky brightness temperature can be written as a function of elevation, el:

$$
T_{\mathrm{b}}(e l)=T_{\mathrm{bg}} \mathrm{e}^{-\tau / \sin (e l)}+T_{\text {eff }}\left(1-\mathrm{e}^{-\tau / \sin (e l)}\right) .
$$

Since we know $\tau, T_{\text {eff }}$ and $e l, T_{\mathrm{b}}(e l)$ can be calculated and we can use the sky as cold load. 
The sky switching measurement yields the difference in brightness temperature measured at altitude $z$ ( $z_{0}$ means ground level and $z_{\mathrm{T}}$ means the height at the tropopause) between two elevations (low, $l$, and high, $h)$ :

$$
\Delta T_{\mathrm{b}}\left(z_{0}\right)=T_{\mathrm{b}}\left(z_{0}, l\right)-T_{\mathrm{b}}\left(z_{0}, h\right) .
$$

The observed $\Delta T_{\mathrm{b}}\left(z_{0}\right)$ can also be expressed in the brightness temperatures as seen from the tropopause, $T_{\mathrm{b}}\left(z_{\mathrm{T}}, e l\right)$, which are attenuated in the troposphere by the $\mathrm{e}^{-\tau / \sin (e l)}$ factor, plus the tropospheric emission, $T_{\text {eff }}\left(\mathrm{e}^{-\tau / \sin (h)}-\mathrm{e}^{-\tau / \sin (l)}\right)$ :

$$
\Delta T_{\mathrm{b}}\left(z_{0}\right)=T_{\mathrm{b}}\left(z_{\mathrm{T}}, l\right) \mathrm{e}^{-\tau / \sin (l)}-T_{\mathrm{b}}\left(z_{\mathrm{T}}, h\right) \mathrm{e}^{-\tau / \sin (h)}+T_{\mathrm{eff}}\left(\mathrm{e}^{-\tau / \sin (h)}-\mathrm{e}^{-\tau / \sin (l)}\right) .
$$

We want to estimate the spectrum as seen from the tropopause, $\Delta T_{\mathrm{b}}\left(z_{\mathrm{T}}\right)=T_{\mathrm{b}}\left(z_{\mathrm{T}}, l\right)-T_{\mathrm{b}}\left(z_{\mathrm{T}}, h\right)$. We therefore have to correct each measured spectrum due to the different opacities in each direction.

Since it is possible to express $T_{\mathrm{b}}\left(z_{\mathrm{T}}, l\right)$ as a linear function of $T_{\mathrm{b}}\left(z_{\mathrm{T}}, h\right)$, due to the low opacity of the middle atmosphere, we can estimate $\Delta T_{\mathrm{b}}\left(z_{\mathrm{T}}\right)=\Delta T_{\mathrm{b}}\left(z_{0}\right) a$, where

$$
a=\frac{\sin (h) / \sin (l)-1}{(\sin (h) / \sin (l)) \mathrm{e}^{-\tau_{\mathrm{T}} / \sin (l)}-A \mathrm{e}^{-\tau_{\mathrm{T}} / \sin (h)}},
$$

$A$ is the attenuation, in the reference beam, due to the small piece of absorber that covers a part of the reference beam.

$$
A=\frac{T_{\text {bal.load }}-\left(T_{\text {eff }}-T_{\mathrm{bg}}\right)\left(\mathrm{e}^{-\tau / \sin (h)}-\mathrm{e}^{-\tau / \sin (l)}\right)+\Delta T_{\mathrm{a}}-T_{\mathrm{b}}\left(z_{0}, h\right)-T_{\text {corr }}}{T_{\text {bal.load }}-T_{\mathrm{b}}\left(z_{0}, h\right)},
$$

where $T_{\text {bal.load }}$ is the physical temperature of the balance load absorber and $T_{\text {corr }}$ is a correction due to the beamwidth of the horn antenna needed for the transformation from antenna to brightness temperature (Section 1.3).

The statistical error from the hot-cold calibration and the correction for the tropospheric opacity originates partly from uncertainties in the elevation angles and in $f_{1}$ and $f_{2}$ (Eqs. (2.2) and (2.3)). The most important factor, however, is the difference between the actual and estimated sky brightness temperature, in the calibration direction. This difference is due to clouds that sometimes make the sky inhomogeneous. These errors are totally less than $5 \%(1 \sigma)$ for each individual measurement ( $\sim 6 \mathrm{~min})$. Systematic errors, for example reflections from the calibration loads and systematic angle measurement errors, are probably also less than $5 \%$.

Several spectra are averaged before a vertical profile is calculated. In this averaged spectrum the statistical errors are almost eliminated and only systematic errors are left.

\subsection{Stand alone calibration}

Once an hour the 22.2-GHz receiver automatically measures the difference spectrum between $20^{\circ}$ and $45^{\circ}$ elevations (sky-dip). If the $21.0 / 31.4-\mathrm{GHz}$ receivers yield no useable data this difference spectrum can be used to estimate the optical depth of the troposphere. We use expression (1.4) and replace $\Delta T_{\mathrm{a}}^{\text {new }}$ with the theoretical difference in brightness temperatures between the two elevations. 


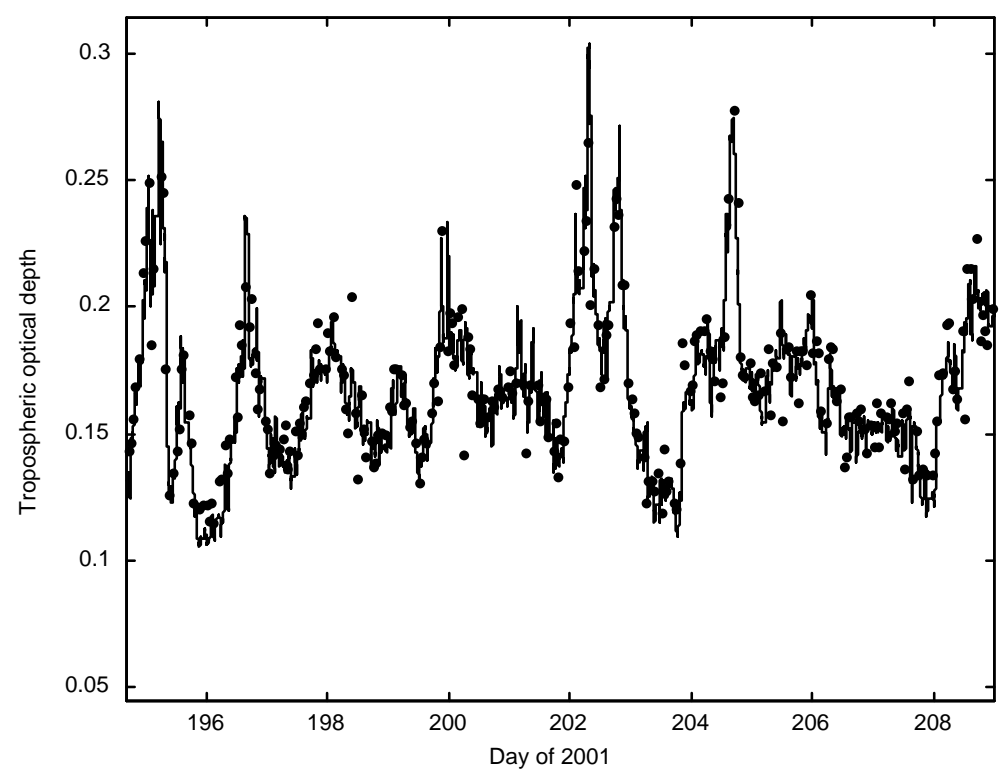

Fig. 7. The tropospheric optical depth calculated from the output of the dual channel radiometer, solid line, and from the 22.2-GHz receiver, dots.

$T_{\text {cold }}$ is estimated from (2.5). Expression (1.4) can then be written as

$$
\left(T_{\text {eff }}-T_{\mathrm{bg}}\right)\left(\mathrm{e}^{-\tau / \sin (h)}-\mathrm{e}^{-\tau / \sin (l)}\right)=\Delta T_{\mathrm{a}}^{\mathrm{old}} \frac{T_{\mathrm{hot}}-\left[T_{\mathrm{bg}} \mathrm{e}^{-\tau / \sin (h)}+T_{\mathrm{eff}}\left(1-\mathrm{e}^{-\tau / \sin (h)}\right)\right]}{T_{\text {hot }}^{\text {def }}-T_{\text {cold }}^{\text {def }}}-T_{\text {corr }},
$$

where $T_{\text {eff }}=0.95 T_{\mathrm{amb}}$ (calculated as in Eq. (2.3) but without the $T_{\mathrm{b}}$ dependence). Since we know everything but $\tau$, the equation can be solved. Fig. 7 shows a comparison between calculated $\tau$ from Eqs. (2.10) and (2.4). The $21 / 31-\mathrm{GHz}$ receiver measures continuously at many elevations and azimuths and equivalent zenith sky brightness temperatures are calculated. Expression (2.10), however, is only valid with the assumption that the sky has the same optical properties in all directions. If the $21 / 31-\mathrm{GHz}$ receiver is turned off for a longer period or the $22-\mathrm{GHz}$ receiver is moved, the sky-dip measurements have to be made at many different elevations to get the same accuracy in the calculated brightness temperatures, as with the $21 / 31-\mathrm{GHz}$ instrument.

By using these sky-dip calibration routines it is actually possible to calculate the brightness temperature of the sky, by using the estimated $\tau$ in Eq. (2.10), with only one calibration load.

The signal elevation angle is adjusted after each spectrum in order to compensate for changes in the tropospheric opacity (see Section 1.7). The strong correlation between the variation of the tropospheric opacity (estimated from the dual-channel radiometer) and the elevation change can be used to estimate the tropospheric absorption in between the calibrations. Fig. 8 shows this correlation.

The statistical and systematic errors are of the same order as described in Section 2.3 (about 5\% each for a $\sim 6$ min measurement). 


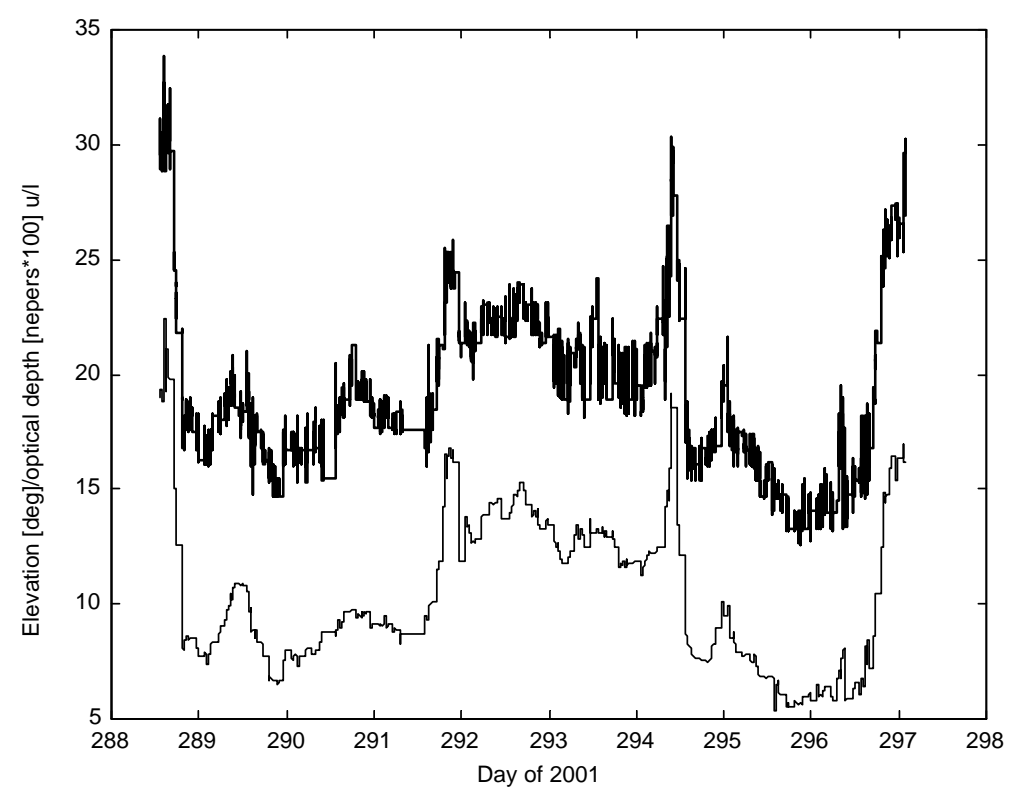

Fig. 8. The variations in the estimated zenith optical depth, from the 21/31-GHz receiver (times 100), lower curve (1), and the variation in the signal elevation $(\mathrm{deg})$, upper curve $(\mathrm{u})$.

This proves that calibration is possible without access to a separate dual-channel water vapour radiometer. It is possible to manage the spectral measurements by doing occasional sky-dips and in between estimating the continuous tropospheric opacity variation from the automatic elevation changes.

\section{Measurement procedures}

We are continuously measuring the sky emission at $22.23 \mathrm{GHz}$ at two different spectrometer configurations. At $16 \mathrm{~h}$ /day the bandwidth $20 \mathrm{MHz}$ (with nominal resolution of $25 \mathrm{kHz}$ ) is used (see Fig. 9) and at $4 \mathrm{~h} /$ day the bandwidth of $320 \mathrm{MHz}$ (with a nominal resolution of $400 \mathrm{kHz}$ ) is used (see Fig. 10). (During the remaining $4 \mathrm{~h}$ the $\mathrm{CO}$ emission at $115.27 \mathrm{GHz}$ is measured using a different frontend receiver but the same backend spectrometer.) The 20-MHz spectra are used to estimate the vertical profile of water vapour between $\sim 45$ and $80 \mathrm{~km}$ altitude. The $320-\mathrm{MHz}$ spectra are used to both investigate the characteristics of the 'baseline ripple' (see Section 4) and try to extend the water vapour vertical profile to altitudes lower than $45 \mathrm{~km}$.

\section{Baseline artefacts}

In the averaged broadband spectrum (Fig. 10) contributions apart from the atmospheric emission are seen. The sum of these contributions is called baseline artefacts and it consists of superpositioned receiver system distortions. These distortions consist of both traces from narrowband gain 


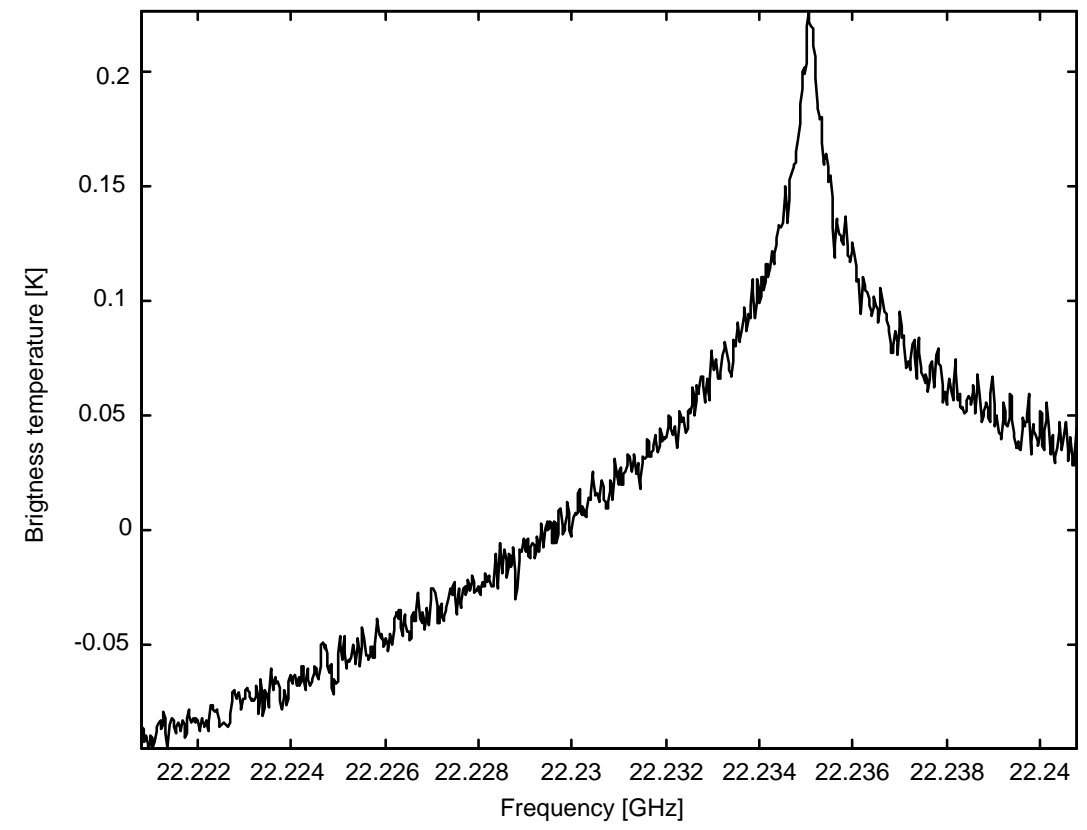

Fig. 9. The average 20-MHz line profile for July 2001. The line centre is offset deliberately in order to register a line wing, which is as long as possible. The spectrum is corrected for tropospheric attenuation but the tropospheric line profile shape is left (seen as an approximately linear slope in this narrow band). The zero point of the brightness temperature scale has been lost due to the balanced differential measurement method, however, the scale factor is correct.

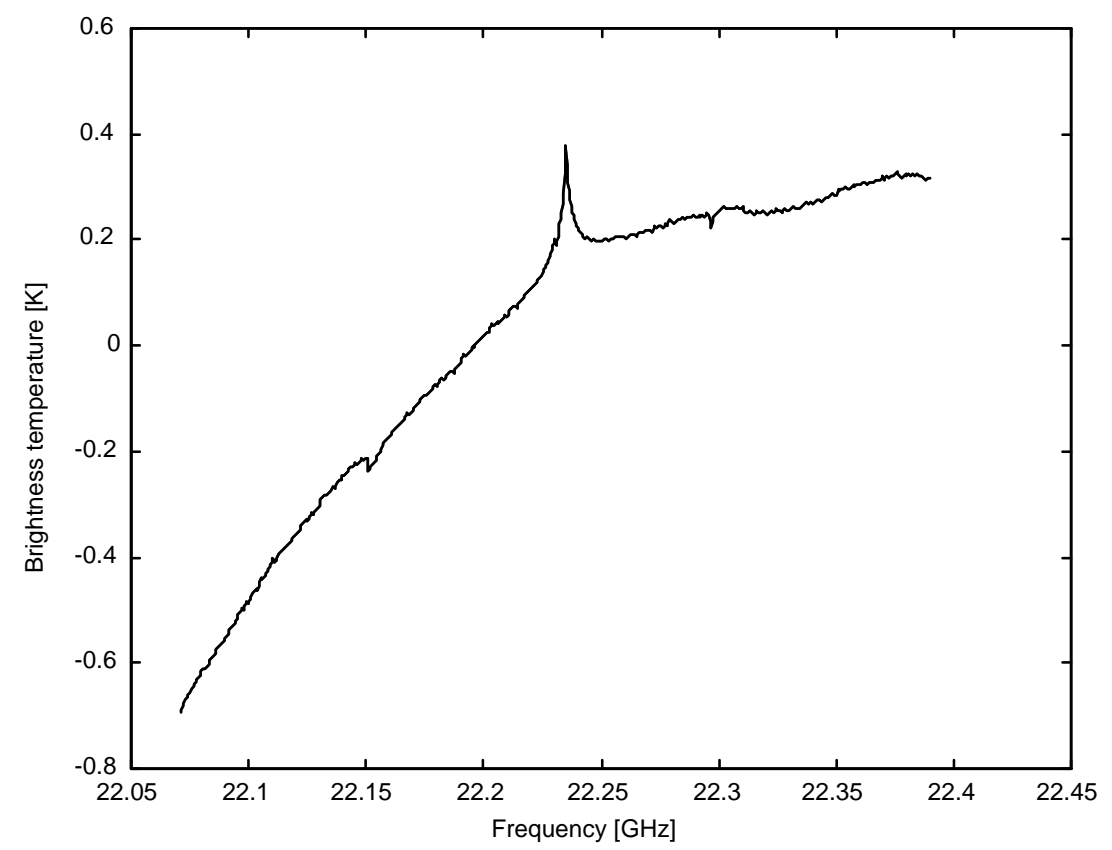

Fig. 10. The average 320-MHz line profile for September 2001. The frequency of maximum tropospheric brightness temperature is higher than the corresponding frequency for the middle atmosphere because of the frequency dependence of the tropospheric pressure broadening. Distortions in the spectrum are commented on in Section 4. Regarding tropospheric attenuation, see Fig. 9. 


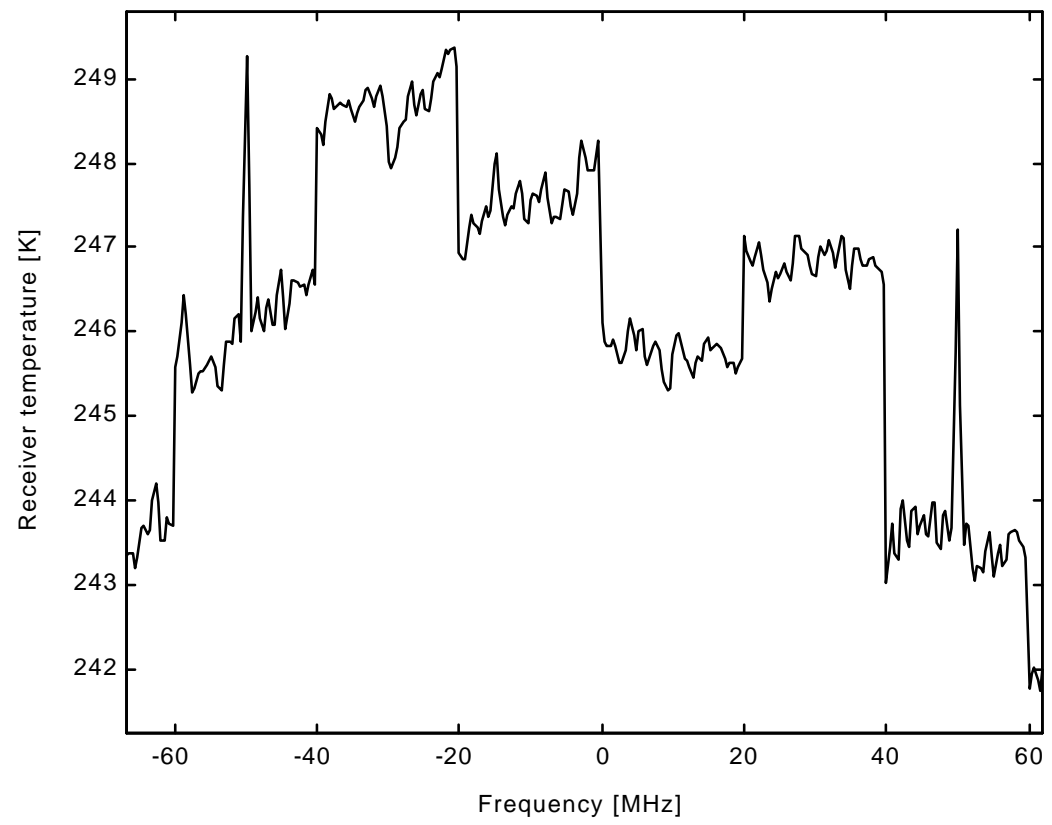

Fig. 11. Measured receiver temperature at $22.2 \mathrm{GHz}$ plotted against offset in frequency from the centre frequency. Note the step at each $20-\mathrm{MHz}$ multiple, which is between two, so-called SSB modules (analogue filters). The 'spikes' at $\pm 50 \mathrm{MHz}$ are signals from oscillators within the receiver. Neither the steps, nor the 'spikes' are hardly seen in the calibrated spectra.

non-linearity variations within the system (for example the small steps at some positions in Fig. 10) and fractions of standing wave patterns arising from reflections in the transmission line. The inversions from spectra to altitude profiles can be improved if these artefacts can be characterised.

\subsection{Gain non-linearity variations}

In radio aeronomy, line radiation often is only a small fraction of the total power received. To be able to remove the influence of the system gain and the receiver temperature Dicke switching is performed (as in our case sky switch, for example).

The normalised calibration difference spectrum (Eq. (1.1)) can be written, if deviations from the nominal gain are included:

$$
\frac{P_{\text {hot }}-P_{\text {cold }}}{P_{\text {cold }}}=\frac{T_{\text {hot }}-T_{\text {cold }}}{T_{\text {cold }}+T_{\text {rec }}}-\frac{\Delta G_{\text {hot }}-\Delta G_{\text {cold }}}{G},
$$

where $\Delta G$ is the difference between the nominal gain and the actual gain. The $\left(\Delta G_{\text {hot }}-\Delta G_{\text {cold }}\right) / G$ factor would vary smoothly from channel to channel if the signal were processed in the spectrometer by identical components in an identical environment. In our spectrometer the IF signal is divided into several $20-\mathrm{MHz}$ bands. Due to minor variations in components and in their physical temperature the factor is not varying smoothly between these bands. This is also seen in the extracted $T_{\text {rec }}$ spectrum as clear steps between the different 20-MHz bands (see Fig. 11). 


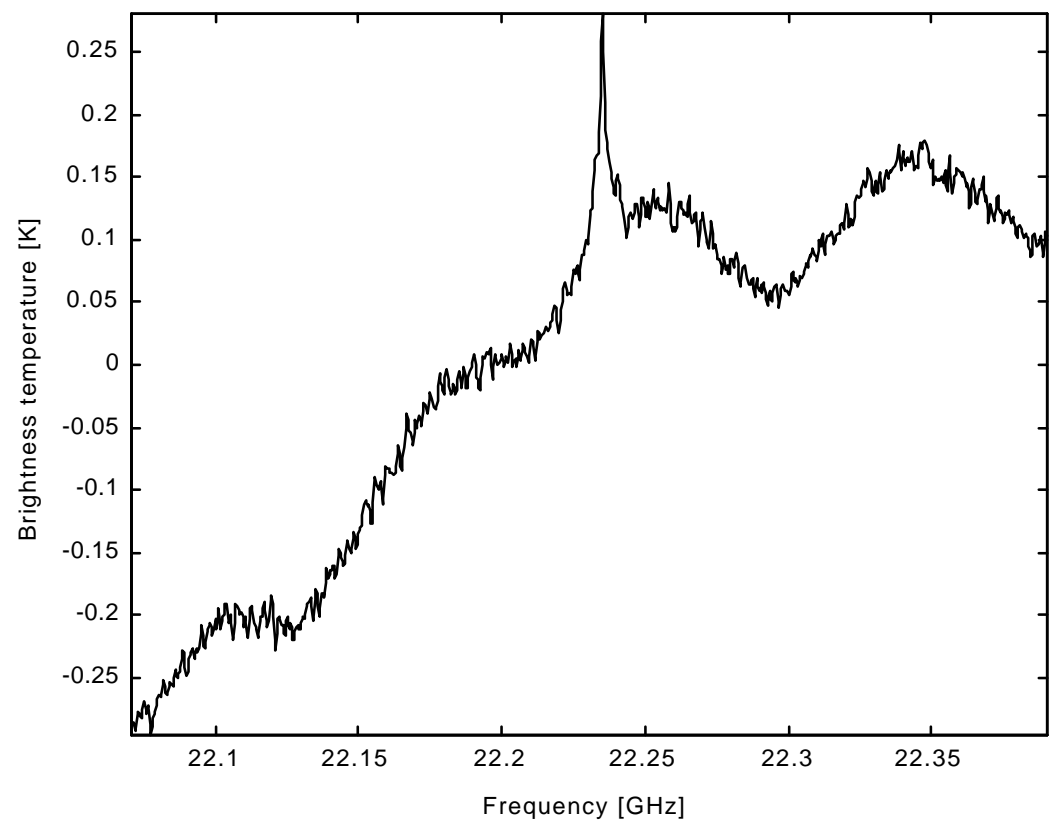

Fig. 12. The narrow peak at $22.235 \mathrm{GHz}$ is the emission from middle atmospheric water vapour. The general slope of the baseline arises from the emission from the tropospheric water vapour. The overlaying sinusoidal pattern is due to reflections at different parts in the transmission line.

Artefacts caused by gain non-linearity problems will be minimised if all components are kept at as stable temperature as possible and if the received emission in the signal and reference directions is kept as similar (balanced) as possible. This could be done in different ways and we have chosen a method similar to that of Nedoluha et al. [2] where a piece of absorber, a balance load, is placed in a part of the reference beam $(\sim 5-10 \%)$ to compensate for the higher intensity in the signal beam by adding blackbody emission (at ambient temperature) to the cold zenith sky brightness temperature. In this method the signal in the reference beam is slightly attenuated (about $5-10 \%$ ) which will decrease the integration time by about $10 \%$ to get the same signal/noise ratio as if no absorber covered part of the reference beam.

\subsection{Standing wave pattern}

The drawback of the 'balance load' method is that some of the noise emitted by the receiver frontend will be reflected in the balance load and will cause 'baseline ripples'. To avoid this, a path length modulator has been mounted to the mirror whose reciprocal movements will cancel out most of the standing waves. Fig. 12 shows an average spectrum from a period when the path length modulator had stopped. The superpositioned sinusoidal pattern arises from reflections at two different positions in the transmission line. From the theory of small reflections, [16], some properties about the reflections can be calculated from the figure. The periodicity, $\Delta f$, of the ripple gives the distance, $\Delta z$, between the reflection positions, $\Delta z=c / 2 \Delta f$ where $c$ is the speed of light. In our case one of the reflection points is the balance load and the other is the waveguide transition at the 
throat of the horn. From the amplitude of the ripple it is possible to estimate the return loss at the balance load. In our case the return loss from the absorber is calculated to be $\sim 24 \mathrm{~dB}$. It is difficult to reach a lower backscattering than this using commercially available absorbing material. We use an anechoic absorber (Eccosorb, CV-3, see Fig. 1). When this material covers most of the beam the return loss can reach $\sim 50 \mathrm{~dB}$ due to the pyramidal shape that cancel out the incident radiation. This effect is decreased when a smaller part of the beam is covered, but this absorber still has better characteristics than other available materials.

\subsection{Baseline characterisation}

In the inversion process a fit to the baseline is made. To make this fit as accurately as possible the baseline characteristics have to be investigated. Polynomials of different degrees have been fitted to different frequency ranges of the spectra. The best baseline characterisation is found when a polynomial fit of at least second degree is made to each $20-\mathrm{MHz}$ interval of the measured spectra. This fit is needed to be able to correct for gain non-linearity variations within each of the analogue filters in the autocorrelator spectrometer. The amplitude of the fitted polynomial varies between 5 and $40 \mathrm{mK}$ within each $20-\mathrm{MHz}$ band where most of these polynomial variations fit the broadband parts of the baseline structure.

\section{Summary}

We have developed a complete, robust and simple radio-aeronomy receiver system to spectrally measure the emission at $22.2 \mathrm{GHz}$ of water vapour in the middle atmosphere. The narrow beamwidth receiver horn in combination with the uncooled HEMT preamplifier makes the receiver system especially simple.

To be able to both measure the water vapour emission at high resolution and to characterise baseline ripple at a broad bandwidth, we have built a hybrid autocorrelator spectrometer. The spectrometer's 800 delay channels can automatically be spread over both narrow and broad bandwidths.

A calibration method has been developed, which uses the sky itself as the calibration cold load. Methods to estimate the sky brightness temperature with and without the help of another instrument have been developed.

\section{Acknowledgements}

Many persons in the technical staff of Onsala Space Observatory have been involved in some aspect of the 22-GHz water vapour receiver, be it in the purchasing of parts, in the construction, in the testing and in the measurements. We would like to mention some of these persons who have been of crucial importance to the project. Peter Hillerström was instrumental in the construction and tuning of the autocorrelation spectrometer. Lars Wennerbäck skillfully manufactured the corrugated horn following the drawings made by Lars Pettersson, who later also measured its properties. Christer Hermansson built many parts of the receiver. Bert Hansson and Magne Hagström played important 
roles in the design work. Lars Lundahl programmed the online computer. Leif Helldner kept-and still keeps - the system up and running and Jamshid Shahrokhi took — and still takes - the data with it.

We would also like to thank Prof. Roy Booth, Director of Onsala Space Observatory, for his unfailing encouragement of the radio-aeronomy project and Joel Elldér for his pioneer work. Prof. Per Simon Kildal, Department of Electromagnetics at Chalmers University of Technology, gave us good advice for the corrugated receiver horn.

We thank the European Council for a grant (No. ENV4-CT97-0515) for building the receiver and for using it in a concerted European measuring campaign (WAter Vapour Experiment, WAVE) within the THESEO programme. We also thank the Swedish Research Council (former Swedish Natural Science Council) for support.

\section{References}

[1] Eriksson P. Analysis and comparison of two linear regularization methods for passive atmospheric observations. J Geophys Res 2000;105(D14):2927-39.

[2] Nedoluha GE, Bevilacqua RM, Gomez RM, Thacker DL, Waltman WB, Pauls TA. Ground-based measurements of water vapor in the middle atmosphere. J Geophys Res 1995;100(D2):2927-39.

[3] Nedoluha GE, Bevilacqua RM, Gomez RM, Thacker DL, Waltman WB, Matthews WA, Hicks BC. Measurements of water vapor in the middle atmosphere and implications for mesospheric transport. J Geophys Res 1996;101(D16):21183-93.

[4] Nedoluha GE, Bevilacqua RM, Gomez RM, Siskind DE, Hicks BC, Russell III JM, Connor BJ. Increases in middle atmospheric water vapor as observed by the Halogen Occultation Experiment and the ground-based water vapor millimeter-wave spectrometer from 1991-1997. J Geophys Res 1998;103(D3):3531-43.

[5] Seele C, Hartogh P. A case study on middle atmospheric water vapor transport during the February 1998 stratospheric warming. Geophys Res Lett 2000;27(20):3309.

[6] Seele C, Hartogh P. Water vapor of the polar middle atmosphere: annual variation and summer mesosphere conditions as observed by ground-based microwave spectroscopy. Geophys Res Lett 2000;26(11):1517.

[7] Emrich AJG. Spectrometers and receiver systems for ground, balloon and satellite based (sub) millimetre radio astronomy. Technical Report, 225, School of Electrical and Computer Engineering, Chalmers University of Technology, Sweden, 1992.

[8] Janssen MA. An introduction to the passive microwave remote sensing of atmospheres. In: Janssen MA, editor. Atmospheric remote sensing by microwave radiometry. New York: Wiley, 1993.

[9] Elgered G, Jarlemark POJ. Ground-based microwave radiometry and long-term observations of atmospheric water vapor. Radio Sci 1998;33(3):707-17.

[10] Jarlemark POJ. Microwave radiometry for studies of variations in atmospheric water vapor and cloud liquid content. Technical Report, 181L, School of Electrical and Computer Engineering, Chalmers University of Technology, Sweden, 1994.

[11] Liebe HJ. Atmospheric spectral properties between 10 and $350 \mathrm{GHz}$ : new laboratory measurements and models. In: Westwater ER, editor. Proceedings of the Specialist Meeting on Microwave Radiometry and Remote Sensing Applications, Wave Propagation Laboratory, NOAA, Boulder, 2000. p. 189-96.

[12] Slobin SD. Microwave noise temperature and attenuation of clouds: statistics of these effects at various sites in the United States, Alaska, and Hawaii. Radio Sci 1982;17(6):1443-54.

[13] Decker MT, Westwater ER, Guiraud FO. Experimental evaluation of ground-based microwave radiometric sensing of atmospheric temperature and water vapor profiles. J Appl Meteorol 1978;17(12):1788-95.

[14] Ingold T, Peter R, Kaempfer N. Weighted mean tropospheric temperature and transmittance determination at millimeter-wave frequencies for ground-based applications. Radio Sci 1998;33(4):905-18.

[15] Peter R, Kaempfer N. Radiometric determination of water vapor and liquid water and its validation with other techniques. J Geophys Res 1992;97(D16):18173-83.

[16] Kildal PS. Foundations of antennas: a unified approach. Lund, Sweden: Studentlitteratur, 2000. 